\title{
Long-Term Survival of Two Patients with Liver Metastases from Advanced Gastric Cancer Treated with Radiofrequency Ablation and Chemotherapy
}

\author{
Sung Jin Oh \\ Department of Surgery, Haeundae Paik Hospital, Inje University College of Medicine, Busan, \\ Republic of Korea
}

\section{Keywords}

Radiofrequency ablation · Liver metastases · Gastric cancer

\begin{abstract}
Liver metastasis from gastric cancer has a very poor prognosis. Herein, we present two cases of liver metastases (synchronous and metachronous) from advanced gastric cancer. In the first case, the patient underwent radical subtotal gastrectomy. Liver metastases occurred 6 months after surgery while the patient was receiving adjuvant chemotherapy, but two hepatic tumors were successfully removed by radiofrequency ablation (RFA). In the second case, liver metastases occurred 15 months after surgery for gastric cancer. The patient also received RFA for one hepatic tumor, and other suspicious metastatic tumors were treated with systemic chemotherapy. Although these case presentations are limited for the efficacy of RFA treatment with systemic chemotherapy for hepatic metastases from gastric cancer, our findings showed long-term survival (overall survival for 108 and 67 months, respectively) of the affected patients, without recurrence. Therefore, we suggest that RFA treatment with systemic chemotherapy could be an effective alternative treatment modality for hepatic metastases from gastric cancer.

(C) 2021 The Author(s).

Published by S. Karger AG, Basel
\end{abstract}

\section{Introduction}

The incidence of synchronous or metachronous liver metastases arising from gastric cancer is approximately $5-14 \%[1,2]$. It is difficult to predict the long-term survival of patients with liver metastases from gastric cancer, with a median survival of less than 6 months, because liver metastasis often represents a hematogenous spread of primary tumors involving 
peritoneal seeding, extensive lymph node metastasis, or direct invasion to adjacent organs [3-5]. However, several studies have reported the efficacy of hepatic resection for metastatic tumors from colorectal cancer and neuroendocrine tumors [6,7], whereas the benefit of hepatic resection for liver metastases from gastric cancer is still controversial $[2,4,8]$.

Current treatment modalities for liver metastasis from gastric cancer, such as surgical resection, radiofrequency ablation (RFA), and hepatic artery infusional chemotherapy, are recommended to improve the survival of these patients with preoperative systemic chemotherapy $[9,10]$. RFA has become a popular alternative to surgery for hepatic metastases owing to its minimal invasiveness and good efficacy. Additionally, the range of RFA applications for hepatic metastases is increasing with the advances in surgical skills and instruments. However, the efficacy and indications of RFA for hepatic metastases are limited and should be accompanied by chemotherapy to improve survival benefits. Owing to the limited reports on the long-term survival of patients with hepatic metastases after surgery for gastric cancer, we herein report two cases of liver metastases from gastric cancer that was successfully treated with a combination of RFA and chemotherapy.

Case 1

A 55-year-old man presented with abdominal discomfort and intermittent abdominal pain. Initial esophagogastroduodenoscopy revealed an ulceroinfiltrative lesion on the lesser curvature of the antrum and the lower body of the stomach. Pathologic examination confirmed the lesion as an adenocarcinoma. Abdominopelvic computed tomography (CT) scan showed an infiltrative well-enhancing mass lesion in the gastric low body to the antrum along the posterior wall and several enlarged lymph nodes in the perigastric area, hepatogastric ligament, and no visible distant metastasis or ascites. The carbohydrate antigen 19-9 level was $41.3 \mathrm{U} / \mathrm{mL}$, and other tumor marker levels were within normal limits. In the field of operation, the gastric tumor showed serosal invasion, and some enlarged lymph nodes were observed around the major vessels. The patient had undergone radical subtotal gastrectomy with Billroth II reconstruction in March 2011. Pathologic reports showed that a diffusely infiltrating mucosal lesion, measuring $7.5 \times 6.0 \mathrm{~cm}$, had been histologically diagnosed as a poorly differentiated adenocarcinoma of the diffuse type. The depth of invasion was serosa (pT4a), and 22 lymph nodes out of 49 regional lymph nodes (pN3b) had metastasized. Lymphatic, venous, and perineural invasion was observed. According to the seventh edition of the AJCC/ UICC classification of gastric cancer, the patient was classified as suffering from stage IIIc cancer. His postoperative course was uneventful, and he was discharged on postoperative day 12. The patient received adjuvant chemotherapy with S-1 (tegafur, gimeracil, and potassium oxonate). Six-month follow-up CT performed after five courses of chemotherapy revealed newly developed multiple, ill-defined, low-attenuated lesions in the liver. Liver MRI showed high signal intensity on T2-weighted images and early hypervascular enhancement, low density on delayed phase, diffuse restriction on diffusion-weighted images, and low signal intensity on delayed phase, which was suggestive of multiple liver metastases $(1.5 \mathrm{~cm}$ in S6 and $1 \mathrm{~cm}$ in S7) (Fig. 1).

The chemotherapy regimen was changed to FOLFOX-6 regimen (2-week cycles of a continuous intravenous infusion of oxaliplatin $100 \mathrm{mg} / \mathrm{m}^{2}$ and leucovorin $100 \mathrm{mg} / \mathrm{m}^{2}$ on day 1, and 5-fluorouracil 2,400 mg/m $\mathrm{m}^{2}$ for $48 \mathrm{~h}$ ). Three month later, follow-up liver MRI showed a stable disease. Subsequently, ultrasonography-guided RFA was performed for two metastatic lesions (S6 and S7) using a 2- to 5-cm exposed big-tip needle. One-month follow-up CT after RFA showed a more involuted state of the RFA sites (S7 and S6), without evidence of local tumor recurrence or delayed complication (Fig. 2), after which the patient underwent 11 consecutive cycles of modified FOLFOX-6.

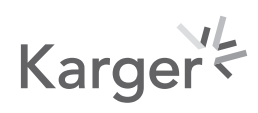


Fig. 1. Liver MRI shows high signal intensity (on T2-weighted images and early hypervascular enhancement, low density on delayed phase, diffuse restriction on diffusion-weighted images, and low signal intensity on delayed phase $(1.5 \mathrm{~cm}$ in $\mathrm{S} 6$ and $1 \mathrm{~cm}$ in S7).
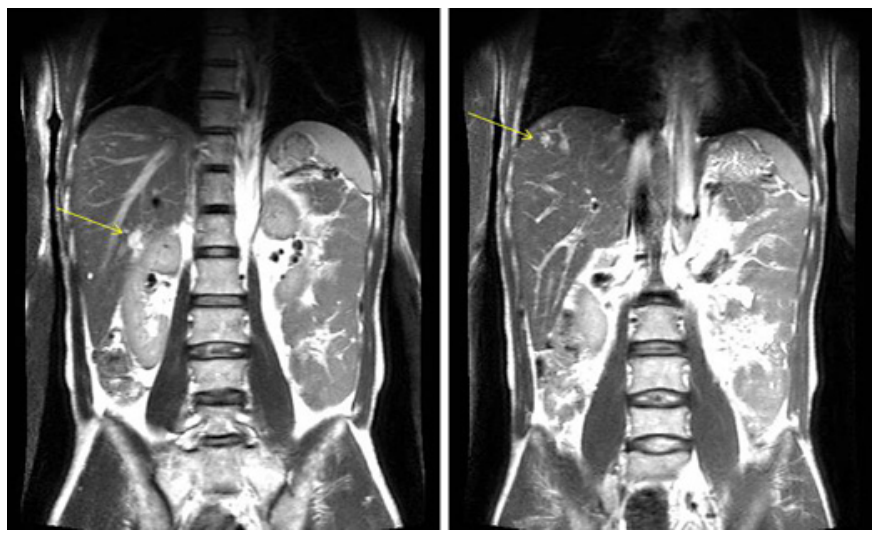

Fig. 2. Liver CT shows a more involuted state of the RFA sites (S7 and S6).
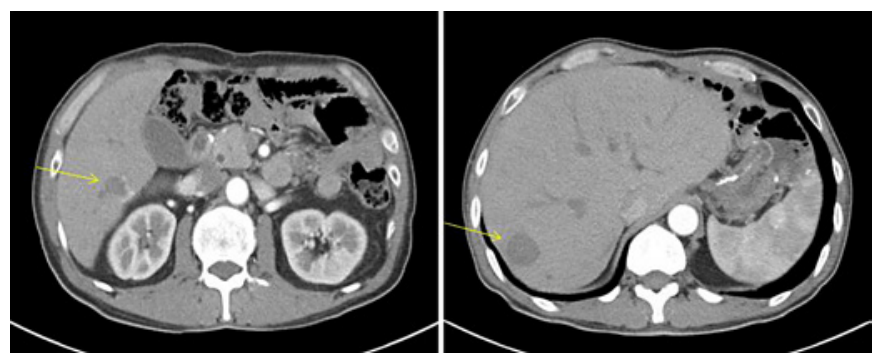

The patient complained of peripheral neuropathy (grade 2), a side effect of chemotherapy. Therefore, intravenous chemotherapy (FOLFOX regimen) was changed to oral doxifluridine, which was tolerated well by the patient. Follow-up CT performed 9 years after surgery revealed no change in the disease status of the patient, compared with the previous CT scan. The patient was still alive without disease progression at the time of writing this article.

Case 2

A 64-year-old woman who had been diagnosed with advanced gastric cancer at a local clinic presented to our hospital complaining of intermittent epigastric pain. Esophagogastroduodenoscopy and 3D CT of the stomach revealed an ulcerofungating mass (measuring approximately $4.3 \mathrm{~cm}$ ) on the greater curvature side of the antrum, with perigastric fat infiltration and several enlarged enhancing lymph nodes in the perigastric area. The patient had undergone radical subtotal gastrectomy with Roux-en-Y reconstruction in September 2014.

Pathologic examination confirmed the presence of a $7.5 \times 7.3 \mathrm{~cm}$ ulcerofungating mass that was histologically diagnosed as a moderately differentiated adenocarcinoma, classified as pT3N3M0 (stage IIIb) according to the seventh edition of the AJCC/UICC classification of gastric cancer. The patient received adjuvant chemotherapy with S-1 (tegafur, gimeracil, and potassium oxonate). Follow-up CT revealed a newly noted low-density hepatic lesion measuring $1.5 \mathrm{~cm}$ at S6 (Fig. 3a). Contrast-enhanced sonography using Sonazoid revealed a thick peripheral rim-like enhancement with a larger volume $(2.1 \times 2 \mathrm{~cm})($ Fig. $3 b)$. Ultrasonography-guided RFA was performed percutaneously for a single metastatic lesion (S6) using a 3-cm exposed cool-tip needle. CT performed 1 month after RFA of the tumor showed interval retraction of the RFA site at S6, without significant complications (Fig. 4a), and a peripheral rim-enhancing low-attenuation lesion in the $\mathrm{S} 4$ dome, which was a probable liver metastasis (Fig. 4b). However, RFA could not be performed, because the suspicious metastatic nodule was close to the patient's heart. The patient received second-line chemotherapy with a 

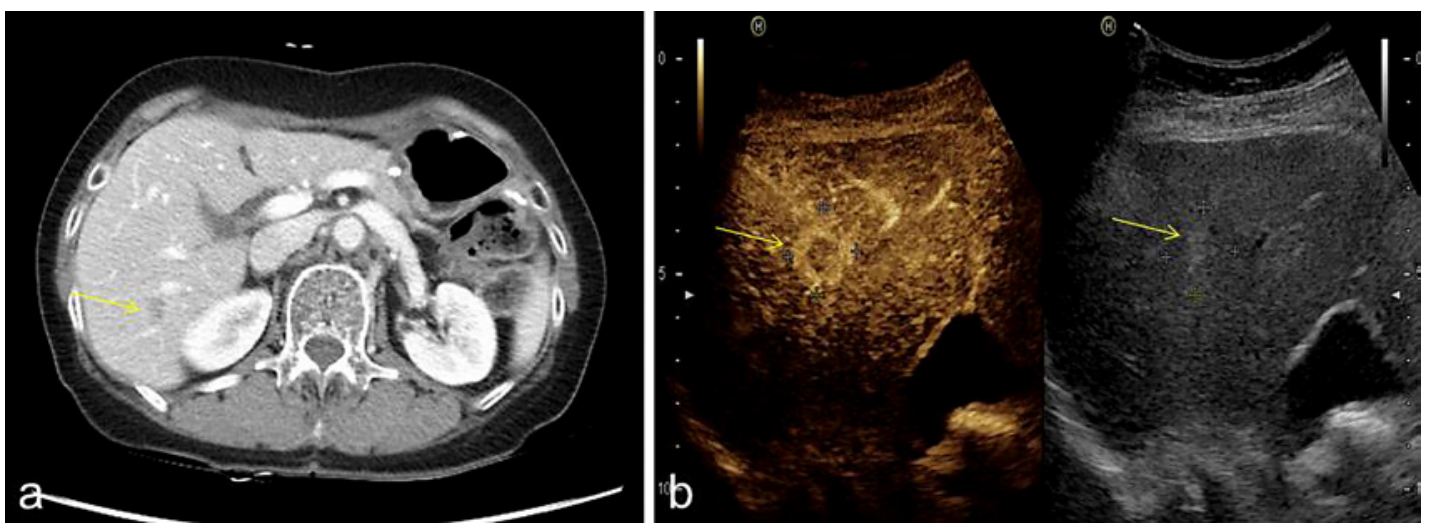

Fig. 3. a Liver CT reveals a newly noted low-density hepatic lesion measuring $1.5 \mathrm{~cm}$ at S6. b Contrast-enhanced sonography using Sonazoid revealed a thick peripheral rim-like enhancement with a larger volume $(2.1 \times 2 \mathrm{~cm})$.

Fig. 4. a Liver CT shows interval retraction of the tumor at RFA site (S6). b Liver CT shows peripheral rim-enhancing low-attenuation lesion in the S4 dome.
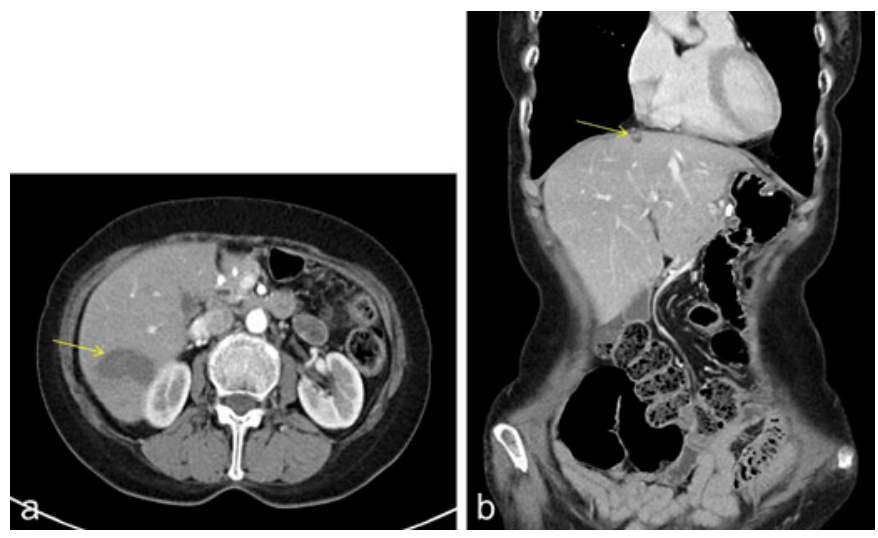

FOLFOX-6 regimen. Follow-up CT of the liver performed 6 months later revealed an interval of more involutional change of the RFA site at S6 without definite evidence of recurrence and no gross interval change of a tiny low-density nodule with decreased peripheral rim enhancement in the S4 dome. Follow-up liver CT performed 50 months after RFA showed shrinkage of RFA zone S6 and no new focal lesion in the liver. The patient was still alive without disease progression at the time of writing this article.

\section{Discussion}

Liver metastasis is one of the most frequent patterns of hematogenous spread in gastric cancer, with a frequency ranging from 5 to $14 \%[1,2,11]$. Hepatic resection is a potentially curable approach for liver metastasis from colorectal cancer, with a 5-year survival rate of $30-50 \%$ [6]. In contrast, patients who undergo hepatic resection for gastric cancer with liver metastasis have typically shown an unsatisfactory survival rate, with highly variable outcomes (15-50\% survival at 1 year and $19 \%$, at 5 years) [4, 8].

RFA has been widely performed as an alternative modality for the treatment of primary and secondary liver tumors that are not candidates for hepatic resection owing to technical advances and effectiveness. However, some factors should be considered to improve the 
efficacy of RFA. The size of the hepatic metastasis is the most important factor for successful complete local ablation. Typically, lesions less than $2.5 \mathrm{~cm}$ in diameter have been reported to have over $90 \%$ chance of being destroyed, whereas approximately $50-70 \%$ of lesions with a diameter of 3.5-5.0 cm can be ablated with RFA. Less than $50 \%$ of lesions measuring more than $5 \mathrm{~cm}$ are likely to be completely treated using RFA. Location is also an influencing factor for achieving complete ablation of metastatic tumors. Complete elimination of tumors located near large vessels (measuring more than $5-10 \mathrm{~mm}$ or those visible on CT) is difficult owing to perfusion-mediated tissue cooling [12]. Tumors near large bile ducts do not tolerate heat, and biliary fistula or strictures would occur after RFA. Another prognostic factor is the number of metastatic lesions. Gannon et al. [10] reported that RFA would be ineffective for more than five metastases in terms of survival benefit. In our cases, the size (less than $2.5 \mathrm{~cm}$ ) and number (less than three) of tumors were suitable for complete RFA for hepatic metastases.

Although systemic chemotherapy has played a pivotal role as a standard treatment for improving the survival of patients with metastatic gastric cancer, the overall survival is just over 12 months and median progression-free survival is approximately 6 months. Fluoropyrimidine (5-fluorouracil, capecitabine, TS-1), anthracycline (epirubicin, doxorubicin), platinum (cisplatin, oxaliplatin), taxane (docetaxel, paclitaxel), and topoisomerase I inhibitor (irinotecan) have been widely used for metastatic gastric cancer [13]. There are several articles about the efficacy and safety of FOLFOX regimens for the treatment of advanced or metastatic gastric cancer $[14,15]$. The FOLFOX- 6 regimen was effective in improving the prognosis of the two patients in this case report, and no severe side effects related to chemotherapy, except for moderate peripheral neuropathy, were observed in either of the patients.

RFA treatment is gradually attracting increasing attention owing to improvements in ablation skills and imaging modalities. However, there have been only a few reports on the long-term survival associated with RFA treatment for patients with liver metastases from gastric cancer. In the current cases, complete long-term remission was achieved using a combination of RFA and systemic chemotherapy. Based on these treatment experiences, we suggest that a combination of RFA with systemic chemotherapy could be an effective treatment option for hepatic metastases from gastric cancer. Further randomized controlled studies are warranted to evaluate the efficacy and safety of RFA plus systemic chemotherapy as a treatment for hepatic metastases from gastric cancer.

\section{Statement of Ethics}

Written informed consent for the publication of the patient's clinical details and images was obtained from the patient.

\section{Disclosure Statement}

The author has no conflicts of interest to declare.

\section{Funding Sources}

The author declared that this study has received no financial support. 


\section{References}

1 Sakamoto Y, Ohyama S, Yamamoto J, Yamada K, Seki M, Ohta K, et al. Surgical resection of liver metastases of gastric cancer: an analysis of a 17-year experience with 22 patients. Surgery. 2003;133(5):507-11.

2 Okano K, Maeba T, Ishimura K, Karasawa Y, Goda F, Wakabayashi H, et al. Hepatic resection for metastatic tumors from gastric cancer. Ann Surg. 2002;235(1):86-91.

3 Zacherl J, Zacherl M, Scheuba C, Steininger R, Wenzl E, Mühlbacher F, et al., Analysis of hepatic resection of metastasis originating from gastric adenocarcinoma. J Gastrointest Surg. 2002;6(5):682-9.

4 Ochiai T, Sasako M, Mizuno S, Kinoshita T, Takayama T, Kosuge T, et al. Hepatic resection for metastatic tumours from gastric cancer: analysis of prognostic factors. Br J Surg. 1994;81(8):1175-8.

5 Bines SD, England G, Deziel DJ, Witt TR, Doolas A, Roseman DL. Synchronous, metachronous, and multiple hepatic resections of liver tumors originating from primary gastric tumors. Surgery. 1993;114(4):799-5

6 Morris EJ, Forman D, Thomas JD, Quirke P, Taylor EF, Fairley L, et al. Surgical management and outcomes of colorectal cancer liver metastases. Br J Surg. 2010;97(7):1110-8.

7 Page AJ, Weiss MJ, Pawlik TM. Surgical management of noncolorectal cancer liver metastases. Cancer. 2014; 120(20):3111-21.

8 Miyazaki M, Itoh H, Nakagawa K, Ambiru S, Shimizu H, Togawa A, et al. Hepatic resection of liver metastases from gastric carcinoma. Am J Gastroenterol. 1997;92(3):490-3.

9 Chen J, Tang Z, Dong X, Gao S, Fang H, Wu D, et al. Radiofrequency ablation for liver metastasis from gastric cancer. Eur J Surg Oncol. 2013;39(7):701-6.

10 Gannon CJ, Curley SA. The role of focal liver ablation in the treatment of unresectable primary and secondary malignant liver tumors. Semin Radiat Oncol. 2005;15(4):265-72.

11 Saiura A, Umekita N, Inoue S, Maeshiro T, Miyamoto S, Matsui Y, et al. Clinicopathological features and outcome of hepatic resection for liver metastasis from gastric cancer. Hepatogastroenterology. 2002;49(46):1062-5.

12 Goldberg SN, Hahn PF, Tanabe KK, Mueller PR, Schima W, Athanasoulis CA, et al. Percutaneous radiofrequency tissue ablation: does perfusion-mediated tissue cooling limit coagulation necrosis? J Vasc Interv Radiol. 1998; 9(1 Pt 1):101-11.

13 Wagner AD, Syn NL, Moehler M, Grothe W, Yong WP, Tai BC, et al. Chemotherapy for advanced gastric cancer. Cochrane Database Syst Rev. 2017;8:Cd004064.

14 Yan D, Dai H. [FOLFOX regimen in the patients with locally advanced or metastatic gastric cancer]. Zhonghua Zhong Liu Za Zhi. 2009;31(3):217-9.

15 Tanaka K, Toyokawa A, Kanemitsu K, Okuda T, Iwasaki T, Ishida T. [A case of gastric carcinoma with lung and liver metastasis successfully treated by mFOLFOX6]. Gan To Kagaku Ryoho. 2010;37(6):1117-20. 